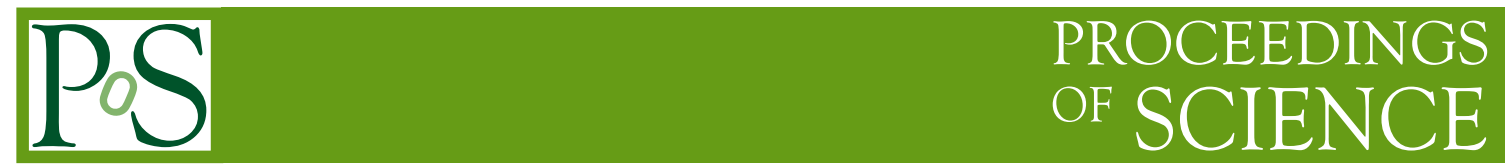

\title{
Cosmology with Wide Field Astronomy
}

\section{Marc Moniez}

Laboratoire de l'Accélérateur Lineaire

E-mail: moniezelal.in2p3.fr

Wide field astronomy has recently produced important results for the dark matter and dark energy problematics. I will summarize the scientific impact of the ongoing wide field surveys and focus on the LSST project (Large Synoptic Survey Telescope). This project will use a $8.4 \mathrm{~m}$ diameter telescope, equipped with a 3.2 Gpixel wide field camera. Cosmological studies is one of his main objectives with a wide capability to address the problematics of the content, the structuration and the expansion history of the Universe, as well as the question of the neutrino mass.

35th International Conference of High Energy Physics - ICHEP2010,

July 22-28, 2010

Paris France

${ }^{*}$ Speaker. 


\section{A brief introduction to cosmology}

The era of cosmological precision measurements started with the developpement of astronomical wide field surveys. Among the precursors, the microlensing surveys, the cosmic microwave background (CMB) missions and the supernovae (SNIa) surveys have contributed to the search for hidden baryons, and to the study of the Universe's expansion. Several problematics of cosmology are directly linked with the particle physics: Understanding the nature of Dark Energy (DE) is one of these fundamental challenges. Many different observations betray the presence of this mysterious energy dominating the Universe; for instance, SNIa distance measurements directly show the accelerated expansion characteristic of DE; the flatness of space indicated by the CMB anisotropies, when combined with large-scale structure (LSS) measurements of the matter content and/or determinations of the Hubble constant, require DE; moreover cross correlations between the CMB anisotropies and LSS show the late-time influence of DE on the growth rate of structure. The so-called concordance model explains the current observations with DE accounting for of order of $75 \%$ of the total energy density of the Universe.

\section{The main cosmological probes}

Modern cosmology uses surveying techniques that have to take into account the expansion of the Universe, which is measured through the wavelength dilatation (redshift $z$ ). Four different definitions of the distance are frequently used, which are linked together through the redshift:

- The comobile distance $d_{c}$ is related to the present position of the object (even observed in a past situation). It follows the Universe's frame which grows with the expansion.

- The luminosity distance $d_{L}=d_{C}(1+z)$ is the distance that would be deduced from the apparent luminosity of an object with known intrinsic luminosity, when assuming a static Euclidian space. It is larger than the comobile distance because galaxies appear fainter than in a static universe.

- The angular distance $d_{A}=d_{C} /(1+z)$ is the distance that would be deduced from the angular size of an object with known dimensions, when assuming a static Euclidian space. Because galaxies were closer at the epoch of the light emission, they appear larger than is a static universe.

- The light travel distance $d_{L T}$ is the propagation time of the photon between its emission and its detection. This distance is used for the lensing studies.

Cosmological probes based on standard candles use objects with known intrinsic luminosities, allowing to estimate a relative luminosity distance. The type Ia supernovae [1] studies enter this category of probes that provide $d_{L}(z)$. Empirical relations where found between the SNIa peak intrinsic luminosity and some of its characteristics (such as extinction time and spectral features); these relations allowed one to improve the definition of a standard candle. Cosmological probes based on standard rulers measure $d_{A}(z)$ of objects or structures with known size. The baryonic acoustic oscillation (BAO) peak observed in the Cosmic Microwave Background (CMB) [2] and 
in the galaxy spatial distributions [3], follows the Universe's expansion (it is imprinted on the socalled Hubble flow); it is considered as a standard ruler because its size is constant in comobile coordinates $\left(d_{C} \sim 150 \mathrm{Mpc}\right)$. Much smaller structures, like galaxies, which are gravitationally bound, do not follow the Hubble flow, because the expansion is locally stopped in the dense regions. In such a case, the size of the ruler does not vary with $z$. The gravitational weak lensing (WL) technique consists in measuring the distorsions of the galaxy orientations due to the gravitational field of the foreground objects. It provides information on the mass distribution, and therefore on the structure formation history [4]. Galaxy cluster counting also provides information on the covolume evolution -connected to $d_{A}(z)$ - and to structure formation.

\section{Large astronomical surveys}

\subsection{Aims and challenges}

An ideal survey should produce a map of galaxies (or larger structures) with a controlled detection efficiency. Such an ideal atlas should contain the angular positions on the sky with the individual redshifts of the objects. Because studies of the Universe's history (BAO, CMB) need a comfortable lever arm in $z$, a deep catalog with precise $z$ determinations is needed. SNIa studies need in addition precise photometric determinations. Studies of the Universe's content with gravitational lensing techniques (WL at large scale, microlensing for local hidden matter searches) also need precise photometric and astrometric determinations plus time resolution. Testing cosmological hypothesis like anisotropy needs large statistical samples and large angular coverages.

All these requirements point to an ideal time resolved deep, wide field, photometric and spectroscopic survey. The following sample of running surveys and projects shows their wide variety: The DES (Dark Energy Survey) is a photometric survey of $5000 \mathrm{deg}^{2}$ with a telescope diameter of $4 \mathrm{~m}$ and a 570 Megapixel digital camera, suited for SN, BAO and WL studies; Pan-STARRS4 comprises 4 telescopes of $1.8 \mathrm{~m}$ diameter with 1.4 billion pixels cameras made of orthogonal transfer CCDs (OTCCDs) for SN searches; JDEM/Euclid is a spatial infrared photometric and spectroscopic project devoted to BAO and WL studies. Among the long term Earth-based projects, BigBOSS is a spectroscopic project using a $2.4 \mathrm{~m}$ telescope with 5000 fiber spectrograph for BAO studies up to $z=3.5$ and SKA (Square Kilometer Array) will be a large digitized multi-purpose radiotelescope that should have a major impact on BAO studies. We will now focus on one of the largest Earth-based survey projects, the Large Synoptic Survey Telescope (LSST).

\subsection{LSST}

The LSST project will use a $8.4 \mathrm{~m}$ diameter telescope, equipped with a 3.2 billion pixels wide field camera, comprised of roughly $2004 K \times 4 K$ CCD sensors, with $10 \mu$ pixels, and fast readout. Its mission will be a multi-pass survey of half of the sky, with 2000 exposures of each 10 square degree patch of the sky spanning six photometric bands $(0.3-1.0 \mu)$ to AB magnitudes 26.5-27. In each year of the survey 250,000 Type Ia supernovae $(z \leq 1)$ will be detected, and prompt alerts will be issued to the international observing community for follow-up spectroscopic observations and observations in other wavelength bands. Surface brightness shapes of over 3 billion galaxies $(z \leq 3)$ will also be measured during the ten-year survey. Precise determination of the Point-Spreadfunction across each image, accurate photometric calibration, and continuous monitoring of system 
performance and observing conditions will lead to unprecedented control of systematic errors. The redshifts will be determined from the six passbands measurements. Images will be acquired every 15 seconds, and image analysis for stringent quality control and detected transient alerts will be generated within 60 seconds. LSST will produce $\sim 120$ Tbytes of raw and preprocessed image and catalog data per full night of observing. The data will be reduced in real time and the resulting images, database, search tools, and software will be made publicly available.

\section{LSST expected performances in cosmology and particle physics}

Figure 1 left shows the expected constraints on the dark energy equation of state $p=w(z)=$ $\left[w_{0}+w_{a} \cdot z /(1+z)\right]$ from the cosmological probes measured by LSST, and their combination [6].

Density fluctuations measured by BAO and weak lensing are also sensitive to the sum of the neutrino masses; indeed massive neutrinos suppress the growth of the matter power spectrum in the small-scale domain, and this effect can be measured by both cosmic shear and BAO measurements (Fig. 1 right). It has been estimated that LSST, in combination with Planck, can constrain the sum of the neutrino masses to $\Sigma m_{v}<0.1 \mathrm{eV}$ and determine the mass hierarchy.
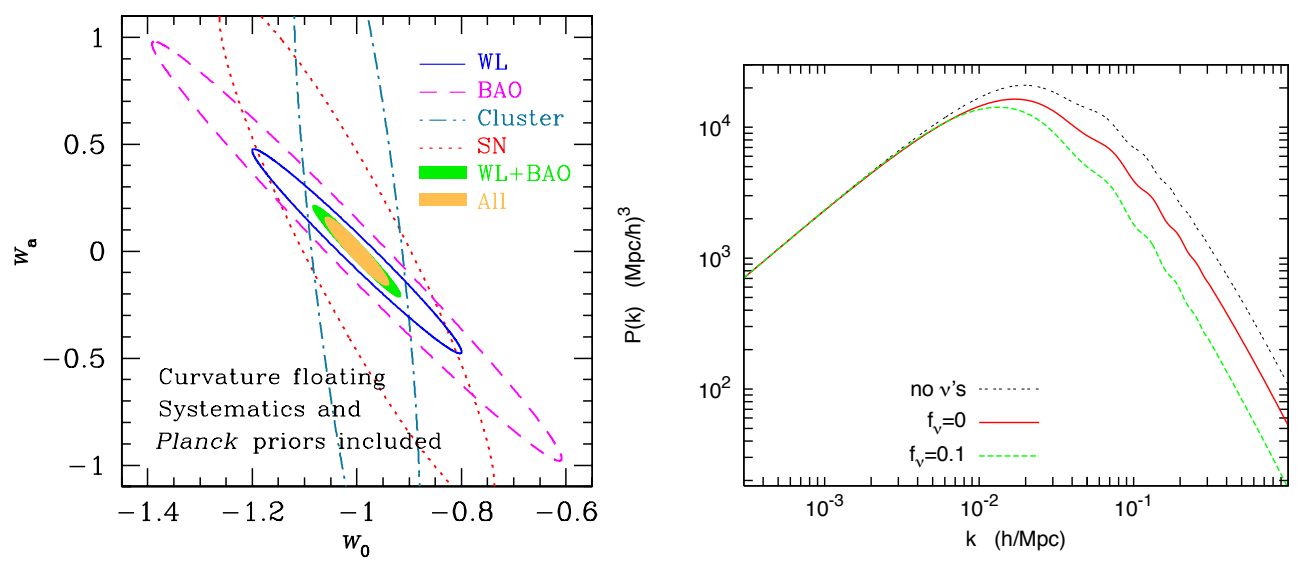

Figure 1: Left: Joint $w_{0}-w_{a}$ constraints from LSST BAO (dashed line), cluster counting (dash-dotted line), supernovae (dotted line), WL (solid line), joint BAO and WL (green shaded area), and all combined (yellow shaded area).

Right: impact of the neutrino masses on the matter power spectrum; $f_{v}$ is the cosmological neutrino density fraction [5].

\section{References}

[1] Astier, P., et al., A\&A, 2006, 441, p.31

[2] Larson, D. et al., to be published in Astrophysical Journal Supplement Series, arXiv:1001.4635v2

[3] Eisenstein, D.J., Zehavi, I., Hogg, D.W., et al. 2005, ApJ, 633, 560

[4] Semboloni, E., et al., A\&A, 2006, 452, p.51

[5] Lesgourgues J. and Pastor, S., Physics Reports, 429, Issue 6, 307-379

[6] LSST Science Book: arXiv:0912.0201 